\title{
Optimization of the Transmitter-Receiver Separation in the Ground-Penetrating Radar
}

\author{
Levent Gürel, Senior Member, IEEE, and Uğur Oğuz
}

\begin{abstract}
The finite-difference time-domain method is applied to simulate three-dimensional subsurface-scattering problems, involving a ground-penetrating-radar (GPR) model consisting of two transmitters and a receiver. The receiving antenna is located in the middle of the two identical transmitters, which are fed $180^{\circ}$ out of phase. This configuration implies the existence of a symmetry plane in the middle of two transmitters and the cancellation of the direct signals coupled from the transmitters at the receiver location. The antenna polarizations and their separations are arbitrary. The transmitter-receiver-transmitter configured GPR model is optimized in terms of the scattered energy observed at the receiver by varying the antenna separation. Many simulation results are used to demonstrate the effects of the antenna separation and the optimal separation encountered for a specific target and GPR scenario.
\end{abstract}

Index Terms-Finite-difference time-domain (FDTD) method, ground-penetrating radar (GPR), perfectly matched layer (PML), subsurface scattering.

\section{INTRODUCTION}

$\mathbf{T}$ HE finite-difference time-domain (FDTD) [4] method has been one of the most popular techniques for the simulations of ground-penetrating-radar (GPR) problems [1]-[3]. The FDTD method has the power of solving problems involving arbitrarily layered media containing arbitrary inhomogeneities. Hence, the method has been preferred for GPR simulations among all differential- and integral-equation-based numerical modeling techniques [5]-[20].

In this paper, the power of the three-dimensional (3-D) FDTD method together with the perfectly matched layer (PML) [21]-[26] absorbing boundary condition is employed to investigate the behavior of a specific GPR configuration. The elements of the GPR problem, as modeled in this study, are depicted in Fig. 1, where the radar unit travels along a linear path above a section of the ground that contains one or possibly more embedded scatterers. The ground is modeled as a homogeneous dielectric half space, the air is modeled as vacuum, and the buried targets are modeled by conducting disks. The disk geometries are accurately modeled using contour-path algorithms [16].

A contemporary method to improve the detection of a subsurface target is to employ GPR configurations with multiple components [27] for the purpose of enriching the content of the

Manuscript received September 7, 2001; revised January 6, 2002. This work was supported in part by Bilkent University under Research Fund EE-01-01, and by the Turkish Academy of Sciences under the Young Scientist Award Program LG/TUBA-GEBIP/2002-1-12.

The authors are with the Department of Electrical and Electronics Engineering, Bilkent University, TR-06533 Bilkent, Ankara, Turkey.

Digital Object Identifier 10.1109/TAP.2003.809873

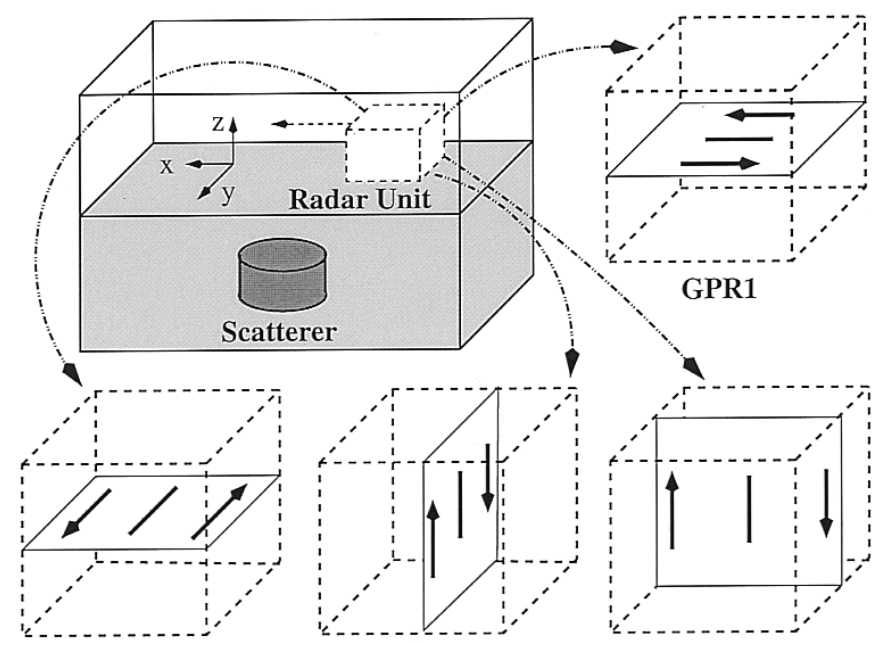

GPR2

GPR3

GPR4

Fig. 1. Geometry of a half-space problem with a buried scatterer. A radar unit travels over the interface at a fixed elevation. The four TRT-configured GPR models, GPR1, GPR2, GPR3, and GPR4 are also depicted. These four GPR models differ in the alignment and polarizations of the three antennas.

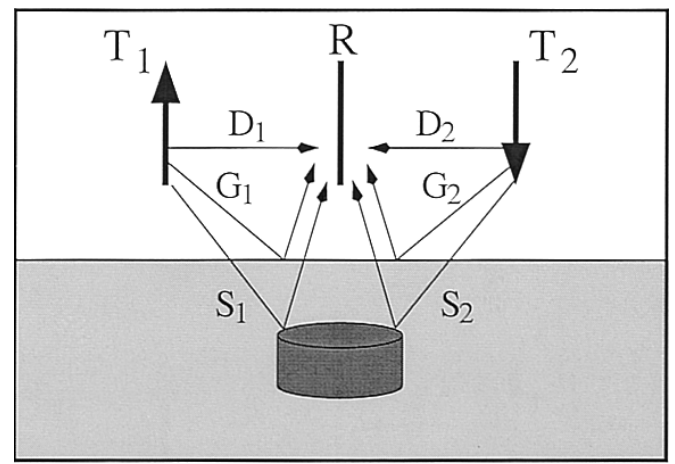

Fig. 2. Basic TRT configuration of the radar unit and the definition of the direct ( $D_{1}$ and $\left.D_{2}\right)$, reflected $\left(G_{1}\right.$ and $\left.G_{2}\right)$, and scattered $\left(S_{1}\right.$ and $\left.S_{2}\right)$ signals.

information received from the target. In this paper, the radar unit consists of two identical transmitting dipoles, modeled by single cells of constant current density [17], and a receiving probe. In a typical GPR unit employing a transmitter and a receiver, the total signal collected by the receiver contains not only the desired scattered signal due to the buried object, but also the direct signal, i.e., the incident field, due to the direct coupling from the transmitter to the receiver. Usually, the direct signal is much larger than the desired signal, rendering the detection of the scattered signal difficult or impossible. In a transmitter-receiver-transmitter (TRT) configuration [12]-[15] illustrated in Fig. 2, feeding the transmitters $\left(T_{1}, T_{2}\right) 180^{\circ}$ out of phase creates a symmetry plane in the middle of these two antennas. Lo- 
cating the receiver $(R)$ on the symmetry plane implies an observed signal totally free of the direct coupling from the transmitters. The coupling from the two transmitters $D_{1}$ and $D_{2}$ cancel each other at the receiver, and moreover, if the ground is homogeneous and the ground-air interface is uniform, the two reflected signals $G_{1}$ and $G_{2}$ also cancel out at the receiver. Even in more realistic situations, where such ideal total cancellations are not possible [18], [19], [28], the TRT configuration is still a powerful way to enhance the detection of buried targets by removing or decreasing the amplitudes of large undesired ("noise") signals. Due to its potential, the TRT-configured GPR [12]-[15] is a subject of further investigation and development.

In this paper, we demonstrate that the detection performance of the TRT-configured GPRs can be significantly improved by optimizing their configuration parameters. In order to support the views presented in this paper, we supply simulation results, which also provide insight to the mechanisms of subsurface scattering and the working principles of GPRs. A sufficient, but modest, amount of results are included here for length considerations. More results are available in [14]. Furthermore, an investigation of the TRT-configured GPRs over randomly heterogeneous grounds and ground-air interfaces with surface roughness is also carried out, and the results are reported elsewhere [18].

The organization of this paper is as follows. Section II describes the common simulation parameters and the basic scenario used to demonstrate the effects of the polarization and the separation of the antennas in a GPR problem. In Section III, the improvement in the performance of a GPR with increasing antenna separation is demonstrated by many simulation results. Section IV argues that an optimum value of antenna separation exists for a specific GPR scenario, and supports this argument with additional simulation results. Section V describes the physical reasons of the observed phenomena in many GPR results by investigating the Poynting vectors both above and under the ground. Finally, Section VI verifies the explanations in the previous section by the help of some additional simulation results, which display the near-field patterns of various GPR units.

\section{Simulation PARAMETERS}

Four GPR models, which differ from each other in the polarization and alignment of the three antennas [13], are used to simulate the scenarios presented in this paper. Fig. 1 displays these four models referred to as GPR1, GPR2, GPR3, and GPR4. GPR1 and GPR2 contain horizontally polarized antennas, whereas GPR 3 and GPR4 consist of vertically polarized antennas. In GPR1 and GPR3, all the antennas are aligned perpendicular to the linear path along which the GPR travels. The configurations of GPR2 and GPR4 are designed such that the antennas are aligned in the direction of the GPR path.

The different alignments and polarizations of GPR models yield variation of the results on an otherwise fixed scenario. Fig. 3 displays four simulation results for a buried conducting disk, obtained with the four TRT-configured GPR models. The physical distance between the receiver and each of the transmitters is set as $5 \mathrm{~mm}$ for all four GPR models. Investigation of Fig. 3(a)-(d) reveals many specific features of the GPR models. First, GPR1 collects the maximum scattered fields compared

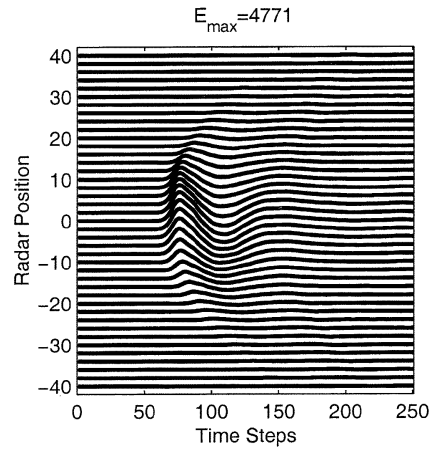

(a)

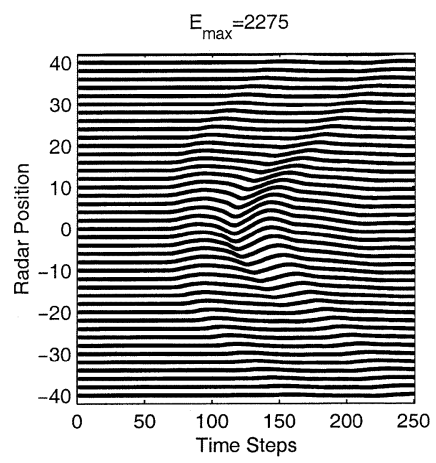

(c)

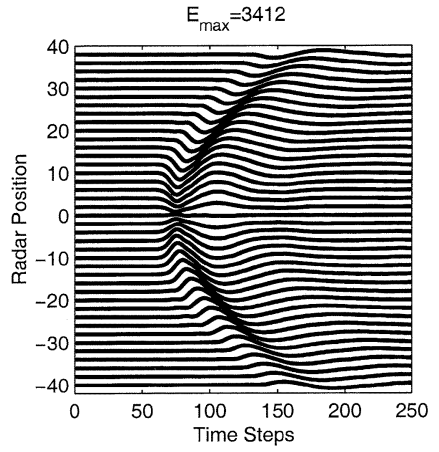

(b)

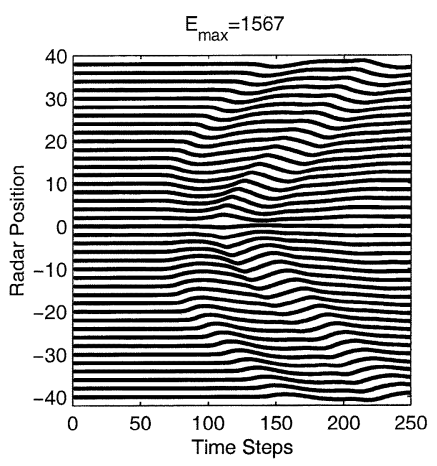

(d)
Fig. 3. Simulation results of a perfectly conducting disk, which is buried $1.25 \mathrm{~cm}$ under the ground, obtained with (a) GPR1, (b) GPR2, (c) GPR3, and (d) GPR4. The ground model has a relative permittivity of $\epsilon_{\text {ground }}=2$, the radius and the height of the disk are 2.5 and $4 \mathrm{~cm}$, respectively.

to the other three models. Second, the responses of GPR2 and GPR4 are similar to each other in the sense that they receive minimum scattered fields when their receivers are close to the center of mass of the conducting disk, while the opposite is true for GPR1 and GPR3. Moreover, GPR2 produces a visible response even when it is far from the target, while GPR1 produces a localized response.

In all simulations, with results presented in Fig. 3 and all through the paper, the transmitting dipoles are modeled as single-cell constant current densities excited by a smooth pulse. The time dependence of this pulse is given by

$$
J(t)=\left[4\left(4 \pi f_{0} t\right)^{3}-\left(4 \pi f_{0} t\right)^{4}\right] e^{-4 \pi f_{0} t}
$$

where $f_{0}$ is the center frequency of the pulse, and $\Delta, \Delta t$ are the spatial and temporal sampling periods, respectively. Due to the constant current density character of the transmitters, the above expression should be divided by a factor of $\Delta^{3}$ to obtain the correct amount of total current [17]. Then, this current term is inserted into the discretized version of the scalar Maxwell's equations at the transmitter location. As an example, the scalar Maxwell's equation and its discretized form governing the electric-field component in the $x$ direction are given by

$$
\frac{\partial E_{x}}{\partial t}=\frac{1}{\epsilon}\left(\frac{\partial H_{z}}{\partial y}-\frac{\partial H_{y}}{\partial z}-\sigma E_{x}-J_{x}\right)
$$

and

$$
E_{x}^{i+(1 / 2), j, k, n}=\frac{2 \epsilon-\sigma \Delta t}{2 \epsilon+\sigma \Delta t} E_{x}^{i+(1 / 2), j, k, n-1}-\frac{2 \Delta t}{\Delta(2 \epsilon+\sigma \Delta t)}
$$




$$
\begin{aligned}
& \text { - }\left(H_{y}^{i+(1 / 2), j, k+(1 / 2), n-(1 / 2)}\right. \\
& -H_{y}^{i+(1 / 2), j, k-(1 / 2), n-(1 / 2)} \\
& \text { - } H_{z}^{i+(1 / 2), j+(1 / 2), k, n-(1 / 2)} \\
& \left.+H_{z}^{i+(1 / 2), j-(1 / 2), k, n-(1 / 2)}\right) \\
& +\frac{2 \Delta t}{(2 \epsilon+\sigma \Delta t)} J_{x}^{i+(1 / 2), j, k, n-(1 / 2)}
\end{aligned}
$$

where $i, j, k$ are the Yee-cell [4] indexes in $x, y$, and $z$ directions, respectively, and $n$ is the time index of the corresponding discrete field variable. For brevity, expressions for the other field components are not given here.

In the simulations with results given in Fig. $3, f_{0}, \Delta$, and $\Delta t$ are set as $1 \mathrm{GHz}, 2.5 \mathrm{~mm}$, and $4.5 \mathrm{ps}$, respectively. However, all other results presented throughout this paper are obtained at a center frequency of $500 \mathrm{MHz}$ and with $\Delta=5 \mathrm{~mm}, \Delta t=9 \mathrm{ps}$.

The receiver is implemented as a small probe, which samples and stores the $x, y$, or $z$ component of the electric field at a specific Yee cell. The polarization of the receiving probe is the same as the transmitting dipoles. A set of observations of the receiver at a stationary point for successive instants of time is called an A-scan. A B-scan is obtained by performing repeated A-scan measurements at discrete points on a linear path. Finally, a C-scan is a collection of the results of multiple B-scan measurements, i.e., A-scan observations made on a two-dimensional (2-D) grid above the ground-air interface [1].

In all of the simulations of the following sections, the target is a conducting disk with $2.5 \mathrm{~cm}$ radius and $4 \mathrm{~cm}$ height, and buried $5 \mathrm{~cm}$ under the ground-air interface.

\section{VARIATION OF THE T-R SEPARATION}

In the previous section, the GPR models contained transmitting and receiving antennas separated by $5 \mathrm{~mm}$. In this section, keeping all of the other simulation parameters fixed, this antenna separation will be modified, and the effect of this variation on the detectability of the buried target will be demonstrated.

Fig. 4 displays the $\mathrm{C}$-scan results of this conducting disk, obtained with four GPR models, all of which contain horizontally polarized antennas but with different separations. The GPR models are moved on a 2-D grid and at each A-scan measurement, the scattered energy is computed as

$$
\text { Energy }=\sum_{n}\left|E^{n}\right|^{2}
$$

where $E^{n}$ is $n$th time sample of the corresponding electric-field variable at that A-scan location. It should be noted that, since the radar units move in two directions, results displayed in Fig. 4 encompass the C-scan measurements of both GPR1 and GPR2 models. Similarly, Fig. 5 displays the C-scan results of both of the GPR models with vertically polarized antennas, i.e., GPR3 and GPR4.

In Fig. 4(a)-(d), the separations of the two transmitters are selected as $1,2,4$, and $8 \mathrm{~cm}$, respectively. Therefore, the receiver, which is located exactly in the middle of the two transmitters, is $0.5,1,2$, and $4 \mathrm{~cm}$ far from the transmitters in Fig. 4(a), (b), (c), and (d), respectively. Similarly, Fig. 5(a)-(d), which displays the C-scan results of both GPR3 and GPR4 models, present the simulation results obtained with $\mathrm{T}-\mathrm{R}$ separation set as $0.5,1,2$, and

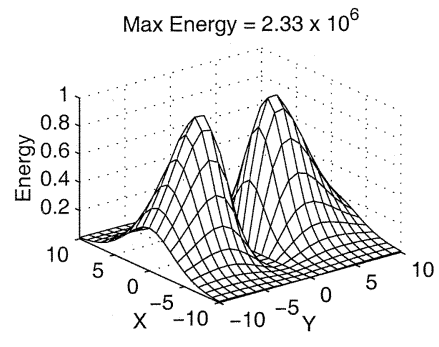

(a)

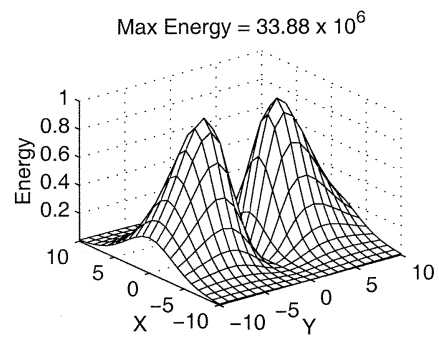

(c)

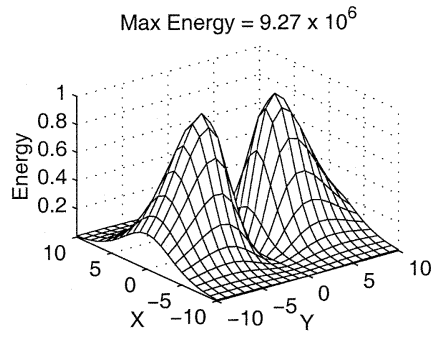

(b)

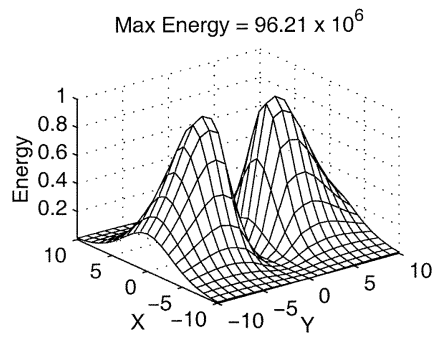

(d)
Fig. 4. C-scan results of a conducting disk, buried $5 \mathrm{~cm}$ under the ground. The GPR unit consists of horizontally polarized antennas, which can simultaneously model both GPR1 and GPR2. The T-R separation is varied from (a) $0.5 \mathrm{~cm}$ to (b) $1 \mathrm{~cm}$, (c) $2 \mathrm{~cm}$, and (d) $4 \mathrm{~cm}$.

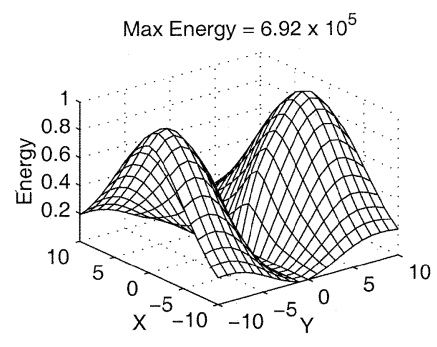

(a)

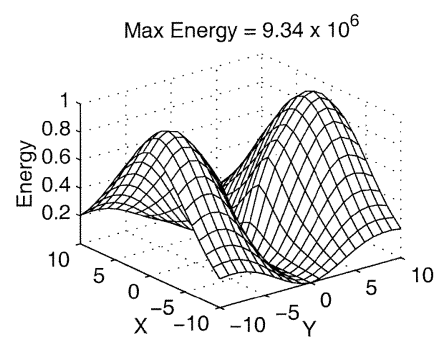

(c)

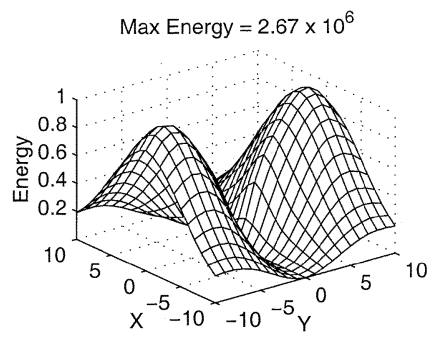

(b)

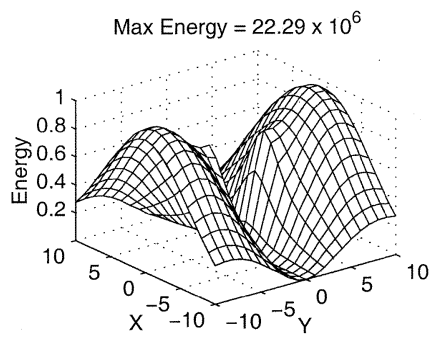

(d)
Fig. 5. C-scan results of a conducting disk, buried $5 \mathrm{~cm}$ under the ground. The GPR unit consists of vertically polarized antennas, which can simultaneously model both GPR 3 and GPR4. The T-R separation is varied from (a) $0.5 \mathrm{~cm}$ to (b) $1 \mathrm{~cm}$, (c) $2 \mathrm{~cm}$, and (d) $4 \mathrm{~cm}$.

$4 \mathrm{~cm}$, respectively. Each constant- $x$ path of the C-scan results in Fig. 4(a)-(d) denote a B-scan observation made by GPR2. Similarly, each constant- $y$ path gives a B-scan result of GPR1. Keeping these features of the C-scan images in mind, careful investigation of Fig. 4(a)-(d) reveals that GPR1 and GPR2 produce B-scan results that vary differently as the position of the target changes with respect to the observation path. GPR1 collects minimum energy when it travels along a path with a projection going through the center of the disk, whereas a maximum is encountered for GPR 2 on the same path. Fig. 5 proves that the same character also holds for GPR3 and GPR4, i.e., on 
the path whose projection passes through the center of the conducting disk, GPR 3 collects minimum and GPR4 collects maximum amount of energy. Note that GPR 1 and GPR 3 have similar symmetry properties with respect to the buried target. The same holds for GPR2 and GPR4.

A more important conclusion revealed from Figs. 4 and 5 is that, for all GPR models, larger amounts of scattered energy are observed on the receiver as the T-R separation increases. Displayed above each of the C-scan plots in Figs. 4(a)-(d) and 5(a)-(d) is the maximum received energy of the corresponding C-scan measurement. Comparison of Fig. 4(a) to (d) reveals the maximum observed energy is raised by almost two orders of magnitude by increasing the T-R separation from 0.5 to $4 \mathrm{~cm}$. A similar increase is demonstrated by Fig. 5(a) and (d) for vertically polarized GPR3 and GPR4 models. The increase of the $\mathrm{T}-\mathrm{R}$ separation not only increases the received energy levels, but also alters the characteristics of the $\mathrm{C}$-scan plots. The reasons for this alteration will be investigated and explained in Section $\mathrm{V}$ in detail.

\section{OPTIMIZATION OF THE T-R SEPARATION}

In the previous section, increasing the T-R separation also increased the observed scattered energy at the receiver location. However, in those simulations, the maximum T-R separation was $4 \mathrm{~cm}$. Since the received scattered energy is likely to decrease to zero as the T-R separation approaches infinity, it is apparent that an optimum value should be encountered as the transmitters and the receiver are taken further away from each other.

In order to demonstrate the existence of this optimum and to find its value, a number of simulations are carried out with models GPR2 and GPR4. For GPR2 and GPR4, the path going through the center of the target is known to produce the maximum scattered energy, as demonstrated in Figs. 4 and 5 of Section III. Similar simulations are not repeated with GPR1 and GPR3 since they have exactly the same maximum energy values. In these simulations, the T-R separation of the GPR models are changed from $1 \mathrm{~cm}$ to $16 \mathrm{~cm}$ in $1-\mathrm{cm}$ steps. Fig. 6(a) displays the simulation results of the same conducting disk, obtained with GPR2 models consisting of horizontally-polarized antennas with 3, 6, 9, and $12 \mathrm{~cm} \mathrm{T-R} \mathrm{separations.} \mathrm{The} \mathrm{simu-}$ lation results obtained with other separation values are not presented here for brevity.

In Fig. 6(a), the horizontal axis denotes the displacement of the receiver from the center of the conducting disk, and the vertical axis denotes the total received energy in that A-scan measurement. Fig. 6(a) demonstrates the existence of an optimum value, since the maximum received energy increases until the $6 \mathrm{~cm}$ value of the T-R separation, and then decreases monotonously for values 9 and $12 \mathrm{~cm}$. A similar conclusion can be drawn from Fig. 6(b), which is obtained with GPR4. The received energy reaches a maximum at 6-cm T-R separation, it stays almost constant for $9 \mathrm{~cm}$, and then slightly decreases at 12-cm T-R separation value. Although both display some local maxima, the responses of GPR2 and GPR4 to the increase of antenna separation are not exactly the same. This difference can be observed from the comparison of Fig. 6(a)-(b). After a par-
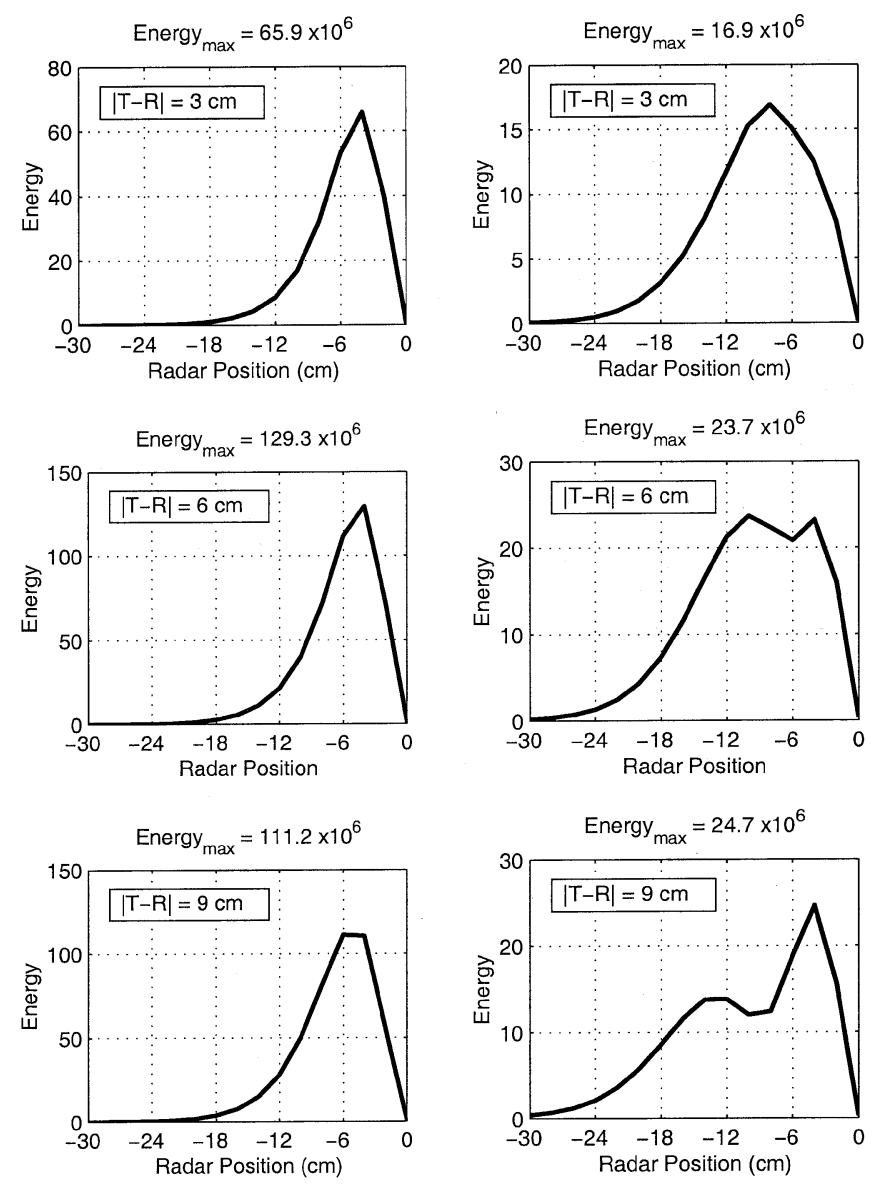

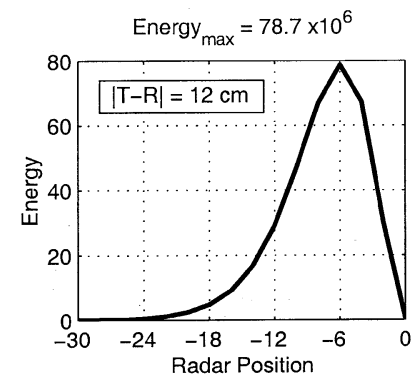

(a)

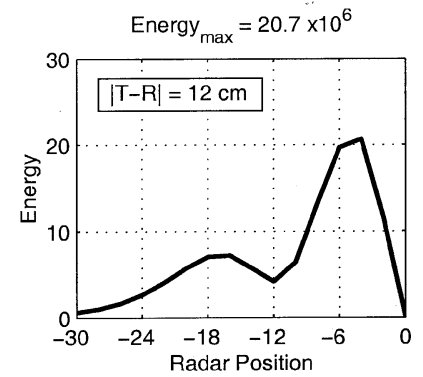

(b)
Fig. 6. B-scan energy plots of a conducting disk. The T-R separation is increased from 3 to $6 \mathrm{~cm}, 9 \mathrm{~cm}$, and $12 \mathrm{~cm}$ for (a) GPR2 and (b) GPR4.

ticular value of the T-R separation, the B-scan results obtained with GPR4 display two local maxima in a single measurement, whereas GPR2 always contains one local maximum. The explanation of these two maxima, or in other words, a minimum in the middle, encountered by GPR4 will be given in the next section.

Fig. 7 displays the maximum received-energy values of GPR2 and GPR4 with respect to the T-R separation. These results are obtained by combining the results of the sixteen simulations performed with each GPR unit. Therefore, Fig. 7 contains the the maximum received-energy values given in the titles of the subplots of Fig. 6(a) and (b). Fig. 7 demonstrates that the received-energy levels start to decrease after a certain T-R separation value for both GPR2 and GPR4. Fig. 7(a) and (b) demonstrates that a T-R separation of $6 \mathrm{~cm}$ gives nearly optimum re- 


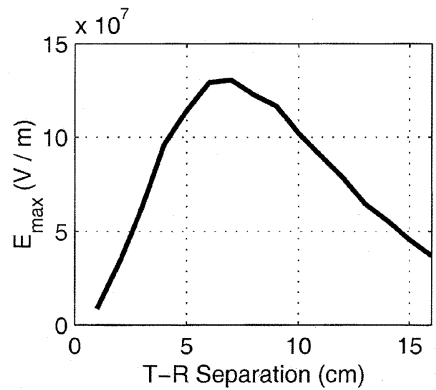

(a)

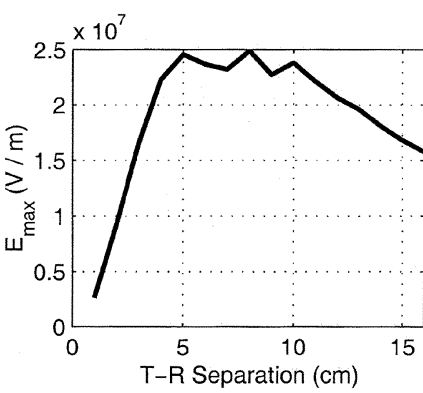

(b)
Fig. 7. Maximum received energy versus T-R separation. The B-scan paths are the same for each value of the $\mathrm{T}-\mathrm{R}$ separation. The maximum energy observed in the B-scan is given for each T-R separation value. The GPR model used is (a) GPR2 and (b) GPR4.

sults for both GPR2 and GPR4, and hence, can be regarded as a good choice for both models.

All the simulation results given in this paper are obtained with the same scatterer buried at the same depth of the ground, and the optimization is performed according to these scenario parameters. The optimum stretch between transmitters and the receiver antennas is subject to change when the parameters of the simulated scenario, such as the depth or the radius of the conducting disk, are altered. However, the rationale of the optimization stays the same, and the choice of the T-R separation is still effective on the observations.

\section{PhysicAl InTERPRETATIONS OF THE OBSERVED PHENOMENA}

In Section IV, the positions of the transmitting and receiving antennas are optimized in terms of the maximum scattered energy observed at the receiver. Moreover, it is shown that GPR2 and GPR4 have different responses to the variation of the T-R separation. GPR4 tends to produce two distinct maxima in a single B-scan measurement as the transmitters and the receiver are taken apart. In this section, the physical explanation of these two peaks will be given. The same conducting disk is used to demonstrate the reasons for this double-peaked character.

Fig. 8(a) and (b) displays the B-scan simulation results obtained with GPR4 of 1- and 16-cm T-R separations, respectively. The arrival times of the scattered signals are retarded as the GPR model with 1-cm T-R separation travels away from the buried target, as depicted in Fig. 8(a). The same retardance cannot be observed in Fig. 8(b), for the GPR model with antennas positioned $16 \mathrm{~cm}$ apart from each other. This difference is due to the variations in the length of the electromagnetic path as the two GPR models travel. The electromagnetic path is the aggregate of individual paths from the transmitters to the target and from the target to the receiver. For closely spaced transmitting and receiving antennas, this total path varies rapidly as the GPR unit travels. However, if the transmitters and the receiver are far from each other, the same amount of displacement of the radar unit does not affect this electromagnetic path at the same rate.

The retardance of the scattered signals as the GPR moves away from the target is not the only difference between Fig. 8(a) and (b). The simulation results of the larger GPR model contains

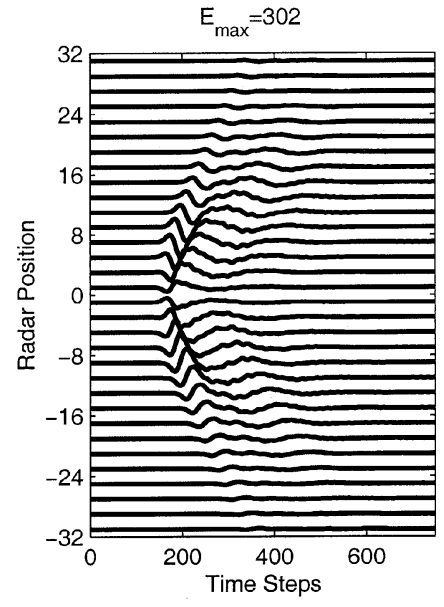

(a)

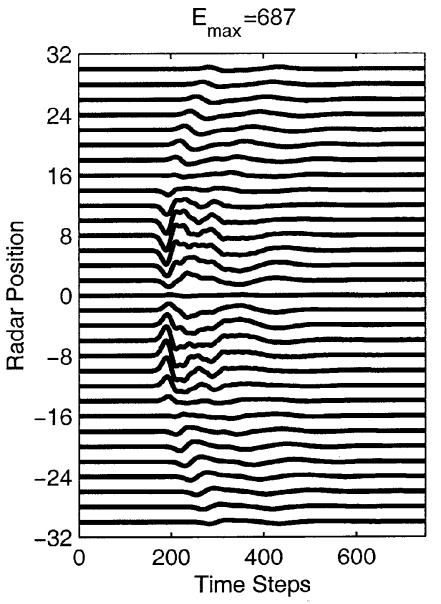

(b)
Fig. 8. B-scan results of the conducting disk with (a) 1-cm-separated and (b) 16-cm-separated GPR4 models. The vertical axis denotes the radar position in terms of cell size $\Delta$, where $\Delta=5 \mathrm{~mm}$, and the horizontal axis denotes the time steps $\Delta t$, where $\Delta t=9$ ps.

minima at two A-scan points symmetric around the center of the conducting disk, as displayed in Fig. 8(b). Such a minimum does not exist in Fig. 8(a), for the case of 1-cm T-R separation. A more careful investigation of Fig. 8(b) reveals that the two minima are observed at the locations, where the transmitters of the GPR model are exactly above the center of the disk. In order to further investigate the reasons for these minima, a series of simulations are performed with the TRT-configured GPR model of 12-cm T-R separation.

Fig. 9(a)-(d) displays the simulation results obtained at particular A-scan locations in a single B-scan measurement. Defining the position of the GPR unit as the position of the receiving probe, four A-scan measurements obtained with GPR4, which are performed $0,6,12$, and $18 \mathrm{~cm}$ far from the center of the buried target, are displayed in Fig. 9(a)-(d), respectively. In each of these figures, the scattered-field Poynting vectors are displayed on a particular plane, which is perpendicular to the ground-air interface and contains the antennas and a vertical slice of the scatterer. In order to obtain these scattered-field Poynting vectors, the scattered fields of the GPR simulation are obtained by

$$
\begin{aligned}
& E^{s}=E-E^{i} \\
& H^{s}=H-H^{i}
\end{aligned}
$$

where $E^{i}$ and $H^{i}$ are the incident electric-field and magneticfield signals. These incident fields are extracted from the results of an extra simulation, performed with a target-free homogeneous ground model. Then, the Poynting vectors in $y$ and $z$ directions are calculated by

$$
\begin{aligned}
& P_{y}=E_{z}^{s} H_{x}^{s}-E_{x}^{s} H_{z}^{s} \\
& P_{z}=E_{x}^{s} H_{y}^{s}-E_{y}^{s} H_{x}^{s}
\end{aligned}
$$

over a fixed $y-z$ plane at every discrete time instant of the simulation. The two components $P_{y}$ and $P_{z}$ constitute a vector on the $y-z$ plane and determine the direction and magnitude of the power flowing out of the scatterer. 


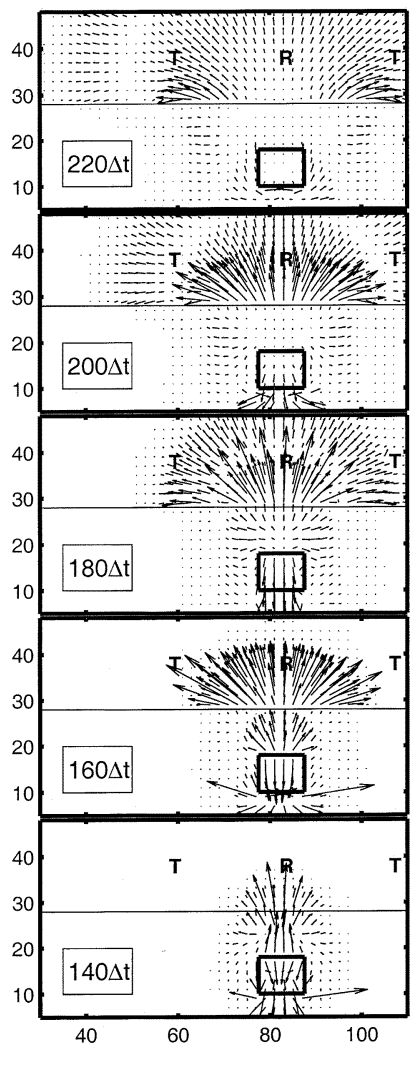

(a)

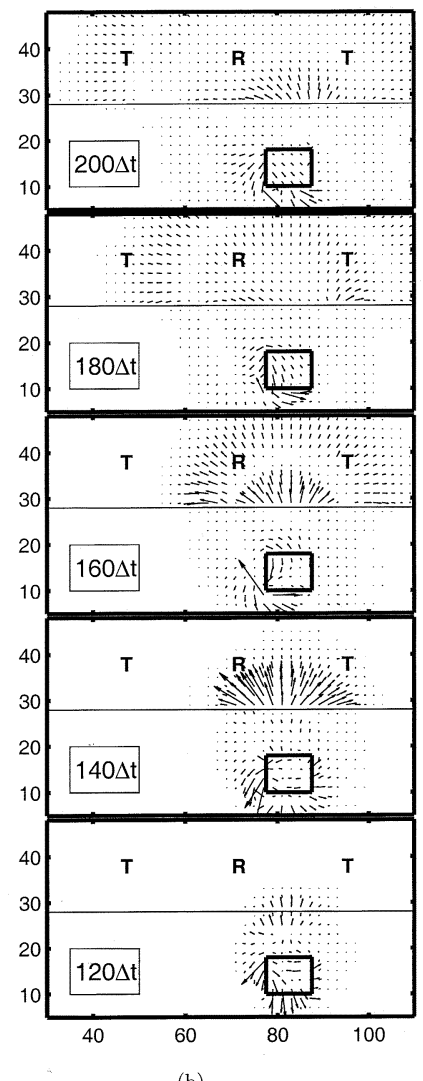

(b)

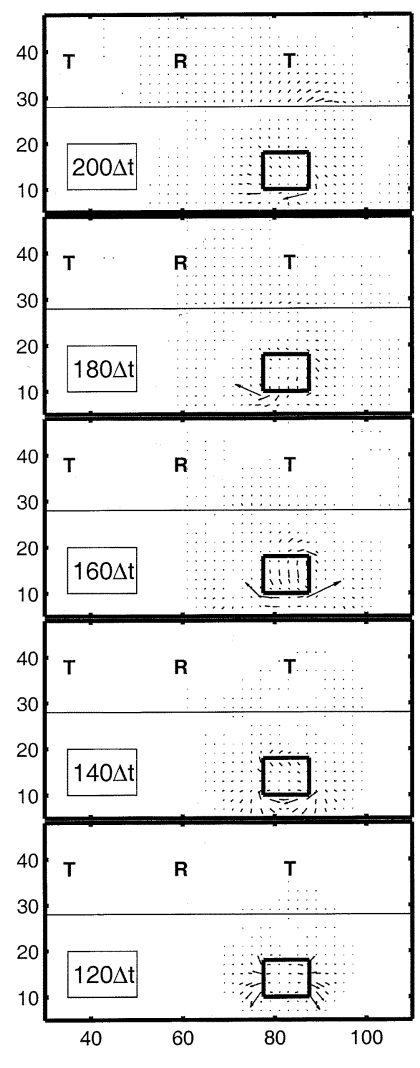

(c)

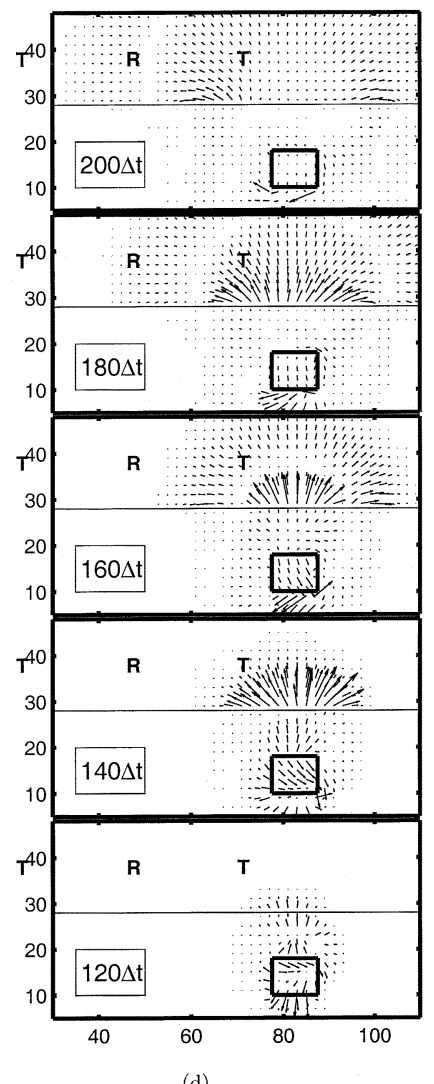

(d)

Fig. 9. Poynting vector plots of 12-cm-separated GPR4, which is located (a) exactly above and (b) at $6 \mathrm{~cm}$ away from the center of the conducting disk. Poynting vector plots of 12-cm-separated GPR4, located (c) $12 \mathrm{~cm}$ and (d) $18 \mathrm{~cm}$ away from the center of the conducting disk.

The sizes of the arrows presented in Fig. 9 do not indicate the exact magnitudes of all Poynting vectors, and they are not all normalized by the same number. In order to illustrate the differences between different A-scans and to be able to display the waves scattering from the target, all arrows are multiplied by a function with $r^{2}$ variation, where $r$ is the distance from a given reference point. In the ground region, the reference point is set as the upper center of the conducting disk since the major reflection from the disk is due to its top face. In the air region, the reference point is the closest point of the ground-air interface to the ground reference, i.e., the point on the interface with the same $x$ and $y$ coordinates as the center of the disk. Moreover, in both regions, a small offset is added to the scaling factors in order to prevent multiplication by zero near the reference points. Finally, since the Poynting vectors in the air region are very small due to the transition from the dielectric medium to the vacuum, all the arrows in the air are multiplied by a factor of 20 to make them visible. Therefore, denoting the reference points in the ground and air regions as $\left(y_{r e f}^{g r}, z_{\text {ref }}^{g r}\right)$ and $\left(y_{r e f}^{a i r}, z_{r e f}^{a i r}\right)$, the arrow plotted at $\left(y_{0}, k_{0}\right)$ is related to the actual Poynting vector at that point by

$$
\tilde{P}=\left[\left(y_{0}-y_{\text {ref }}^{g r}\right)^{2}+\left(z_{0}-z_{\text {ref }}^{g r}\right)^{2}+0.01\right] P
$$

in the ground region, and by

$$
\tilde{P}=20\left[\left(y_{0}-y_{\text {ref }}^{\text {air }}\right)^{2}+\left(z_{0}-z_{\text {ref }}^{\text {air }}\right)^{2}+0.01\right] P
$$

in the air region. Fig. 9(a)-(d) also depicts the antennas, the ground-air interface, and the buried scatterer. For each A-scan, five different time instants are displayed, in order to illustrate the propagation of the waves.

Fig. 9(a) presents the Poynting vectors recorded in the A-scan measurement where the receiver is exactly above the center of the buried disk. The five time instants displayed in this figure are $t=140 \Delta t, t=160 \Delta t, t=180 \Delta t, t=200 \Delta t$, and $t=220 \Delta t$. Fig. 9(a) demonstrates that a large amount of scattered power reaches the receiving antenna. However, note that the power incident on the receiver flows in the $z$ direction. Since the receiving probe of GPR4 samples and stores only the $z$ component of the electric field, and since the direction of power flow is perpendicular to the electric and magnetic fields, GPR4 records zero scattered signals at this A-scan measurement.

The next A-scan measurement shown is the case where the receiver is $6 \mathrm{~cm}$ away from the center of the scatterer. Fig. 9(b) displays the Poynting vectors observed at this position, where the target is in the middle of the receiver and one of the transmitters. Fig. 9(b) demonstrates that the receiver collects a considerable amount of power at this position. Since the power incident on the receiver does not flow solely in the $z$ direction, the GPR unit records large scattered signals at this position. In fact, this A-scan position is the point that GPR4 collects maximum scattered energy. It is also possible to explain this maximum by specular reflection from the top face of the conducting disk since the angle of incidence (from one transmitter to the target) is equal to the angle of reflection (from the target to the receiver) due to the symmetry of the receiver and one of the transmitters with respect to the scatterer. 

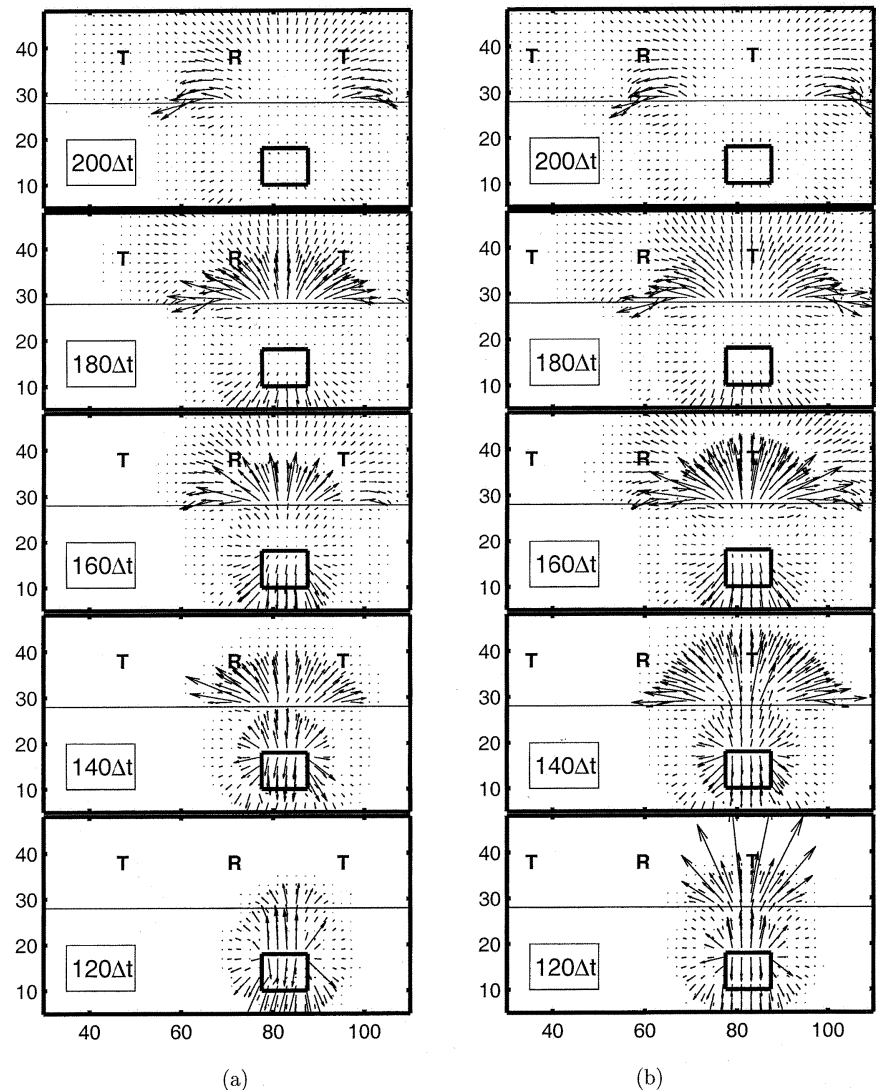

Fig. 10. Poynting vector plots of 12-cm-separated GPR2, which is located (a) $6 \mathrm{~cm}$, and (b) $12 \mathrm{~cm}$ away from the center of the conducting disk.

In Fig. 9(c), where one of the transmitters is exactly above the center of the disk, the flow of scattered power back to the air region is minimal. This minimum was also observed in the energy diagrams of Fig. 6(b). The reason for this minimum is that the $z$-polarized transmitting dipole radiates minimum power toward its axis. Fig. 9(d) presents the Poynting vectors observed when the center (receiver) of the $z$-polarized radar unit is $18 \mathrm{~cm}$ away from the center of the target. At this position, although the receiver is moved further away from the target compared to Fig. 9(c), the GPR receiver collects larger scattered energy. This is due to the larger radiation by the transmitter in the direction of the buried target.

In order to further demonstrate that the polarization of the transmitting dipole is the reason for observing less power in Fig. 9(c) compared to Fig. 9(d), Fig. 10 displays the Poynting vectors obtained at these two positions of the radar unit, only this time GPR2 is used instead of GPR4. Fig. 10(a) displays the results obtained when the target is in the middle of the receiver and a transmitter, while Fig. 10(b) displays the results observed when one of the transmitters is above the center of the buried disk. Unlike the GPR4 scenario in Fig. 9(c), the GPR2 scenario with the same configuration does not result in minimum scattered (and received) energy. Fig. 10(a) and (b) reveals that the distance to the target is the main factor that affects the scattered power recorded by GPR2. This feature is also demonstrated by the B-scan energy diagrams shown in Fig. 6(a), where the received energy makes a single peak and monotonously decreases afterward, as the GPR unit is moved farther away from the target.
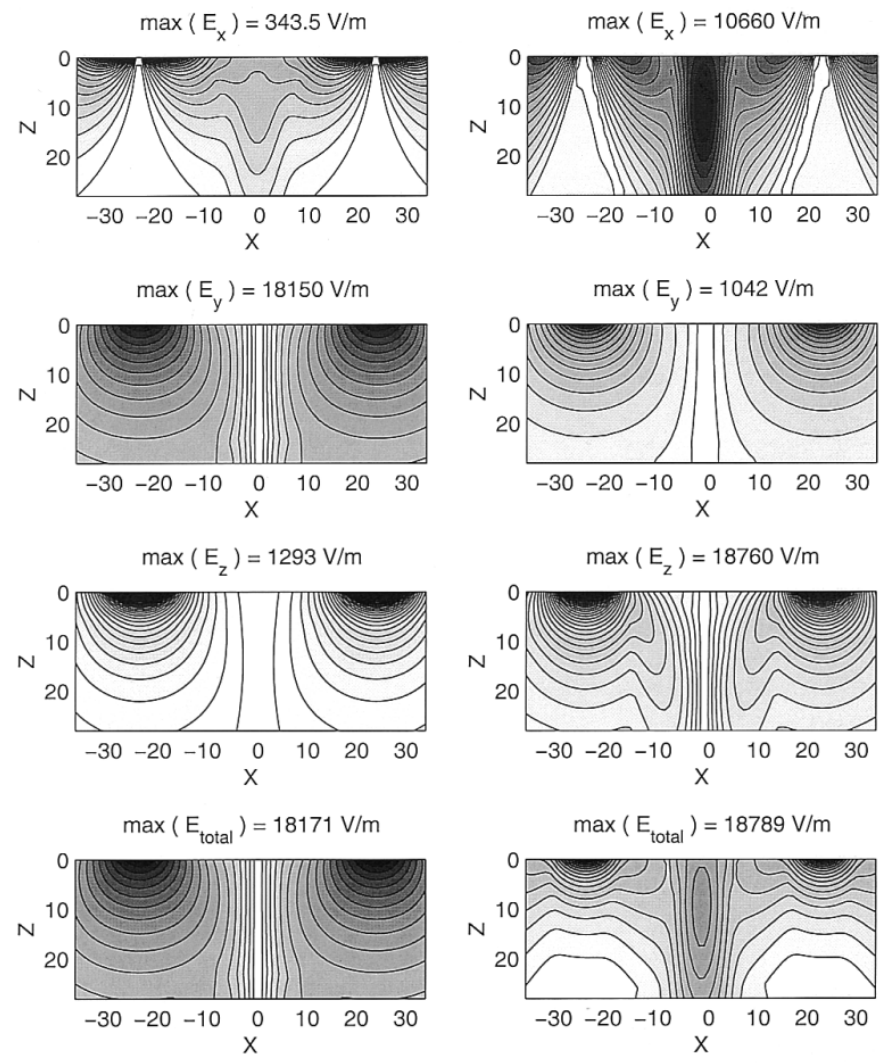

(a)

(b)

Fig. 11. Near-zone $x, y, z$, and total-field patterns of (a) GPR2 and (b) GPR4. The T-R separation is $12 \mathrm{~cm}$.

\section{NEAR-FIELD RADIATION PATTERNS OF GPR UNITS}

The reasoning stated in the previous section on the effects of increasing the T-R separation can also be observed from the near-field radiation patterns of the GPR models. Performing GPR simulations above a homogeneous ground model, i.e., free of any scatterers, the electric-field signals are recorded on a plane under the ground-air interface. This observation plane is almost identical to the plane on which Poynting vectors were displayed in the previous section. It is perpendicular to the ground-air interface, and its extension contains the three antennas of the GPR unit. In order to extract the patterns of the GPR models, the maximum values of the electric-field variables are observed at each point on this plane by

$$
E_{\max }=\max _{n} E^{n}
$$

where $n$ is the discretized time index. In this manner, four different pattern plots can be obtained from a single simulation by observing the maximum values of the $x, y$, and $z$ components, and by calculating the value of the total electric field therefrom. These radiation patterns are first obtained for TRT-configured GPR models of 12-cm T-R separations. Fig. 11(a) and (b) displays the $x, y, z$, and total-field patterns of GPR2 and GPR4, respectively. Fig. 11(a) demonstrates that the total-field pattern of GPR2 is dominated by the $y$ component of the electric field, since the polarization of the transmitting dipoles are in the $y$ direction. Additionally, there exists a null at the symmetry plane of the $y$-component pattern of GPR2, as displayed in Fig. 11(a). 

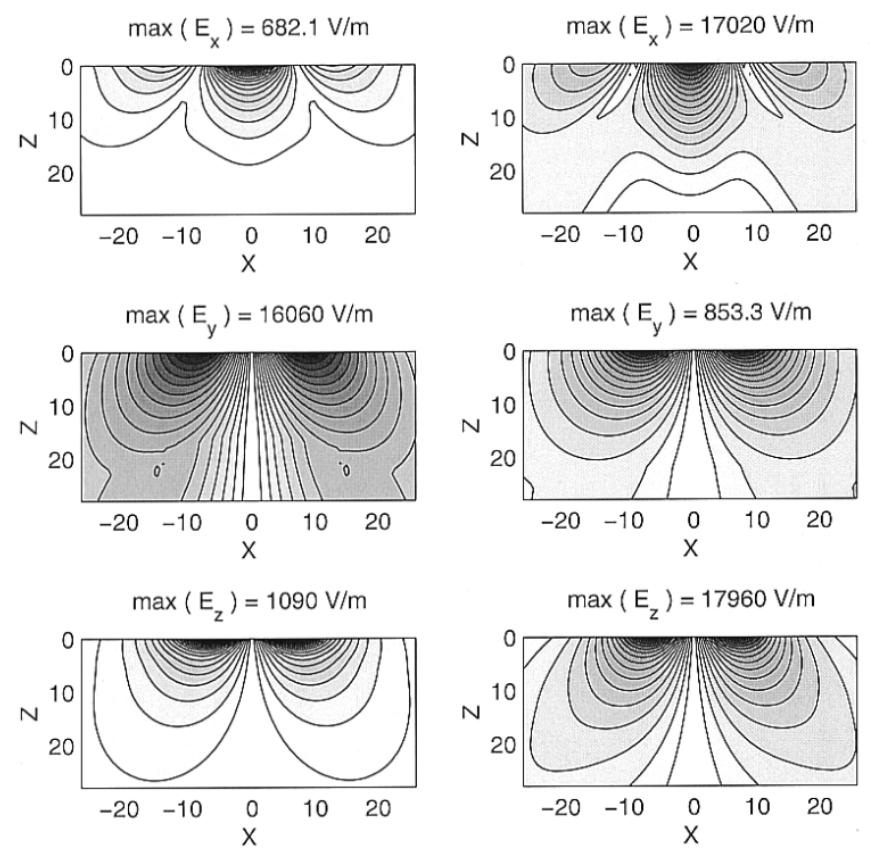

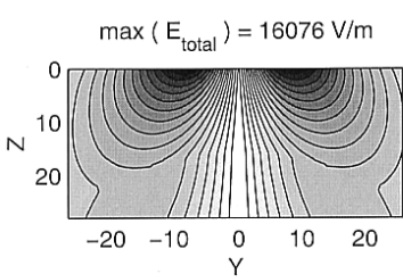

(a)

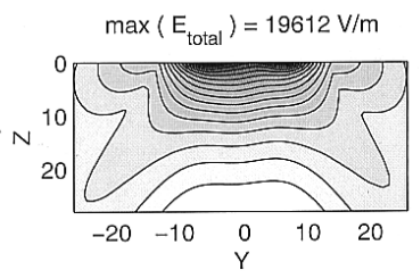

(b)
Fig. 12. Near-zone $x, y, z$, and total-field patterns of (a) GPR2 and (b) GPR4. The T-R separation is $3 \mathrm{~cm}$.

Another noteworthy feature of this pattern is that the main lobe has a single maximum, which explains the single peak in the corresponding energy plot shown in Fig. 6(a). Similarly, the double peak observed for GPR4 in Fig. 6(b) can be explained by noting that the total-field pattern in Fig. 11(b) has significantly large side lobes in addition to the main lobe.

Comparison of Fig. 11(b) to Fig. 11(a) reveals the basic differences between GPR2 and GPR4. For example, comparison of the $y$-polarized pattern of GPR2 displayed in Fig. 11(a) to the $z$-polarized pattern of GPR4 in Fig. 11(b) demonstrates that the former contains darker contours in the main lobe of the pattern. This darkness implies that the fields penetrating into the ground are stronger for GPR2. This is in agreement with the maximum energy values given in the titles of $\mathrm{C}$-scan results for GPR2 and GPR4 in Figs. 4 and 5. GPR1 and GPR2 generate waves with larger amplitudes penetrating into the ground, and hence, larger scattered fields are received by their receivers compared to GPR3 and GPR4.

Not all T-R separation values yield a second energy peak in a GPR4 B-scan. In Fig. 6(b), the GPR4 configurations with smaller antenna separations produced energy plots similar to GPR2. This similarity is demonstrated by Fig. 12(b), where a single-lobe character is displayed for the $z$-polarized pattern of GPR4 with 3-cm T-R separation.

\section{CONCLUDING REMARKS}

In this paper, the GPR has been simulated with the 3-D FDTD method. The ground is modeled as a homogeneous dielectric medium and the air is modeled as vacuum. The buried target is selected as a conducting disk with a particular radius, height, and depth. The GPR is modeled by two identical transmitting dipoles and a receiving probe in between. By using this TRT configuration, the direct coupling from the transmitters to the receiver has been eliminated. Four different TRT configurations are used in the simulations presented in this paper.

In the TRT configuration, the separation between the antennas affects the amount of received scattered energy. In previous work [13], closely spaced antennas were used. It is demonstrated that increasing the antenna separation enhances the reception of scattered fields by increasing the energy of the scattered fields incident on the receiver. However, this increase is not monotonic, and after a particular separation of the antennas, which can be regarded as the optimum value, the received energy starts to decrease rapidly.

For some GPR configurations, the search for an optimum value of antenna separation peculiarly results in double peaks instead of a single maximum that readily reveals the optimum value. The reasons for these peculiarities are investigated by the use of Poynting vector plots obtained from the FDTD simulations. By means of this powerful tool, it is observed that for one of the GPR models with vertically polarized antennas, the transmitters apply a minimum of fields on the target when they are above the scatterer. This phenomenon yields an unexpected minimum in the B-scan results that is explained with the use of Poynting-vector plots and near-field radiation patterns of GPRs.

The results presented here are sufficient to prove the claims and to explain the observations made in this paper. Nonetheless, we present elsewhere [14] an extensive amount of useful results in various formats, which are not included here for brevity.

The optimization of the antenna separation is of major importance, especially for scenarios governing heterogeneous ground models. In such problems, optimization of the antenna placement implies the maximization of the signal-to-noise ratio of the raw signals at the receiver. The use of TRT-configured GPRs in the presence of heterogeneous grounds is studied elsewhere [18], [19].

\section{REFERENCES}

[1] D. J. Daniels, Surface-Penetrating Radar. London: IEE Press, 1996.

[2] A. C. Dubey, J. F. Harvey, and J. T. Broach, Eds., Detection and Remediation Technologies for Mines and Minelike Targets III Proceedings. Orlando, FL: SPIE, Apr. 1998.

[3] GPR'98, Seventh International Conference on Ground-Penetrating Radar. Lawrence, KS: Univ. Kansas, 1998.

[4] K. S. Yee, "Numerical solution of initial boundary value problems involving Maxwell's equations in isotropic media," IEEE Trans. Antennas Propagat., vol. 14, pp. 302-307, May 1966.

[5] M. Moghaddam, W. C. Chew, B. Anderson, E. J. Yannakakis, and Q. H. Liu, "Computation of transient electromagnetic waves in inhomogeneous media," Radio Sci., vol. 26, no. 1, pp. 265-273, Jan.-Feb. 1991.

[6] M. Moghaddam, E. J. Yannakakis, W. C. Chew, and C. Randall, "Modeling of the subsurface interface radar," J. Electromag. Waves Applicat., vol. 5, no. 1, pp. 17-39, 1991.

[7] K. Demarest, R. Plumb, and Z. Huang, "FDTD modeling of scatterers in stratified media,” IEEE Trans. Antennas Propagat., vol. 43, Oct. 1995. 
[8] J. M. Bourgeois and G. S. Smith, "A fully three-dimensional simulation of a ground-penetrating radar: FDTD theory compared with experiment," IEEE Trans. Geosci. Remote Sensing, vol. 34, pp. 36-44, Jan. 1996.

[9] U. Oğuz and L. Gürel, "Subsurface-scattering calculations via the 3D FDTD method employing PML ABC for layered media," in 1997 IEEE AP-S Int. Symp. URSI Radio Science Meeting, Montréal, Canada, July 1997.

[10] F. L. Teixeria, W. C. Chew, M. Straka, and M. L. Oristaglio, "Finite-difference time-domain simulation of ground-penetrating radar on dispersive, inhomogeneous, and conductive soils," IEEE Trans. Geosci. Remote Sensing, vol. 36, pp. 1928-1937, Nov. 1998.

[11] J. M. Bourgeois and G. S. Smith, "A complete electromagnetic simulation of the separated-aperture sensor for detecting buried land mines," IEEE Trans. Antennas Propagat., vol. 46, pp. 1419-1426, Oct. 1998.

[12] P. Luneau and G. Y. Delisle, "Underground target probing using FDTD," in 1996 IEEE AP-S Int. Symp. URSI Radio Science Meeting, Baltimore, MD, July 1996, pp. 1664-1667.

[13] L. Gürel and U. Oğuz, "Three-dimensional FDTD modeling of a groundpenetrating radar," IEEE Trans. Geosci. Remote Sensing, vol. 38, pp. $1513-1521$, July 2000.

[14] U. Oğuz and L. Gürel. (2001, Mar.) The effects of the antenna separation and polarization on the simulation results of transmitter-receiver-transmitter-configured ground-penetrating radars. [Online] (June 6, 2001). Available: http://www.cem.bilkent.edu.tr/gpr/

[15] L. Gürel and U. Oğuz, "Transmitter-receiver-transmitter configurations of the ground-penetrating radar," Radio Sci., vol. 37, no. 3, pp. 51-57, Nov. 2001.

[16] T. G. Jurgens, A. Taflove, K. Umashankar, and T. G. Moore, "Finite-difference time-domain modeling of curved surfaces," IEEE Trans. Antennas Propagat., vol. 40, pp. 357-366, Apr. 1992.

[17] D. N. Buechler, D. H. Roper, C. H. Durney, and D. A. Christensen, "Modeling sources in the FDTD formulation and their use in quantifying source and boundary condition errors," IEEE Trans. Microwave Theory Tech., vol. 43, pp. 810-814, Apr. 1995.

[18] L. Gürel and U. Oğuz, "Transmitter-receiver-transmitter-configured ground-penetrating radars over randomly heterogeneous ground models," Radio Sci., vol. 37, Nov.-Dec. 2002.

[19] U. Oğuz and L. Gürel, "Simulation of TRT-configured ground-penetrating radars over heterogeneous grounds," in Proc. 2001 IEEE AP-S Int. Symp. USNC/URSI National Radio Science Meeting, Boston, MA, July 2001.

[20] U. Oğuz and L. Gürel, "Modeling of ground-penetrating-radar antennas with shields and simulated absorbers," IEEE Trans. Antennas Propagat., vol. 49, pp. 1560-1567, Nov. 2001.

[21] J. P. Berenger, "A perfectly matched layer for the absorption of electromagnetic waves," J. Comput. Phys., pp. 185-200, Oct. 1994.

[22] W. C. Chew and W. H. Weedon, "A 3D perfectly matched medium from modified Maxwell's equations with stretched coordinates," Microwave Opt. Technol. Lett., vol. 7, no. 13, pp. 599-604, Sept. 1994.

[23] D. S. Katz, E. T. Thiele, and A. Taflove, "Validation and extension to three dimensions of the Berenger PML absorbing boundary condition for FD-TD meshes," IEEE Microwave Guided Wave Lett., vol. 4, pp. 268-271, Aug. 1995.

[24] J. B. Verdu, R. Gillard, K. Moustadir, and J. Citerne, "An extension of the PML technique to the FDTD analysis of multilayer planar circuits and antennas," Microwave Opt. Technol. Lett., vol. 10, no. 6, pp. 323-327, Dec. 1995.

[25] J. Fang and Z. Wu, "Generalized perfectly matched layer for the absorption of propagating and evanescent waves in lossless and lossy media," IEEE Trans. Microwave Theory Tech., vol. 44, pp. 2216-2222, Dec. 1996.
[26] D. Johnson, C. Furse, and A. Tripp, "Application and optimization of the perfectly matched layer boundary condition for geophysical simulations," Microwave Opt. Technol. Lett., vol. 25, no. 4, pp. 253-255, May 2000 .

[27] F. Lehmann, D. E. Boerner, K. Holliger, and A. G. Green, "Multicomponent georadar data: Some important implications for data acquisition and processing," Geophysi., vol. 65, no. 5, pp. 1542-1552, Sept.-Oct. 2000.

[28] U. Oğuz and L. Gürel, "Simulations of ground-penetrating radars over lossy and heterogeneous grounds," IEEE Trans. Geosci. Remote Sensing, vol. 39, pp. 1190-1197, June 2001.

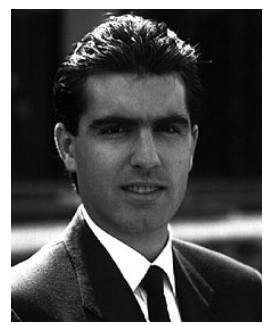

Levent Gürel (S'87-M'92-SM'97) was born in İzmir, Turkey, in 1964. He received the B.Sc. degree from the Middle East Technical University (METU), Ankara, Turkey, in 1986, and the M.S. and Ph.D. degrees from the University of Illinois at Urbana-Champaign (UIUC) in 1988 and 1991, respectively, all in electrical engineering.

He joined the Thomas J. Watson Research Center of the International Business Machines (IBM) Corporation, Yorktown Heights, NY, in 1991, where he worked as a Research Staff Member on the electromagnetic compatibility (EMC) problems related to electronic packaging, on the use of microwave processes in the manufacturing and testing of electronic circuits, and on the development of fast solvers for interconnect modeling. Since 1994, he has been a faculty member with the Department of Electrical and Electronics Engineering, Bilkent University, Ankara. Turkey. He was awarded the title of Associate Professor by the Institute of Higher Education, Ankara, in 1993. He was a Visiting Associate Professor at the Center for Computational Electromagnetics (CCEM), UIUC for one semester in 1997. His research interests include the development of fast algorithms for computational electromagnetics (CEM) and the application thereof to scattering and radiation problems involving large and complicated scatterers, antennas and radars, frequency-selective surfaces, and high-speed electronic circuits. He is also interested in the theoretical and computational aspects of electromagnetic compatibility and interference analyses. Ground-penetrating radars and other subsurface-scattering applications are also among his current research interests. He has published several papers in IEEE journals.

Dr. Gürel is currently serving as the Chairman of the AP/MTT/ED/EMC Chapter of the IEEE Turkey Section. He is a recipient of the Science Scholarship of the Turkish Academy of Sciences (TUBA) and presently serves as Associate Editor of Radio Science. He has actively attended numerous IEEE symposia and served as a reviewer for various IEEE journals. He is a Member of the Electromagnetics Academy.

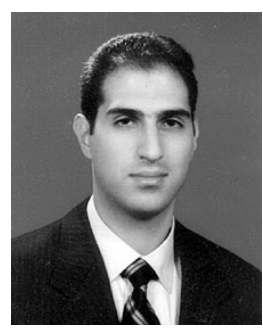

Uğur Oğuz was born in Ankara, Turkey, in 1973. He received the B.Sc. and M.Sc. degrees, both in electrical engineering, from Bilkent University, Ankara, Turkey, in 1994 and 1997, respectively.

From August 1997 to October 1998, he served in the Turkish Army, working as a Database Manager. From November 1998 to June 2002, he was a Research Engineer with the Department of Electrical and Electronics Engineering, Bilkent University. His research interests include time-domain methods in computational electromagnetics and their applications to geophysical problems. Currently, he is a Systems Engineer in Aselsan A.S., Ankara, Turkey. 